\title{
Design of a Clamping Device for the Free Curve Welding
}

\author{
X. Huang ${ }^{1,2}$, J. Wang ${ }^{1}$ and R. XIAO ${ }^{2}$ \\ ${ }^{1}$ School of Mechanical and Electrical Engineering, North China University of \\ Technology, Beijing, China \\ ${ }^{2}$ Institute of Laser Engineering Beijing, Beijing University of Technology, China \\ marchbupt@gmail.com
}

\begin{abstract}
Developing a clamping device fixed directly on a multi-degree of freedom robot manipulator to solve the problem of easy deformation of sheet metal plates in three dimensions free curve is still very challenging. This work describes the conceptual design, the mechanical design, and the design of control system of a new clamping device for the free curve welding. The basic function of the clamping device is to provide a stable and adjustable mechanical pressure which is generated by a compressed conventional spring to a sheet plate in the welding process. Following the concept design stage, the mechanical design is performed, aimed at effectively controlling the deformation of sheet metal and eliminating the gap between the plates in the welding process. Finally, the control methodology is put forward. Thanks to the proposed modular construction and the use of a conventional spring, the device shows good performances. The developed clamping device can also be extended to other applications in surface manufacturing such as spray painting on space free curve.
\end{abstract}

Keywords: Clamping Device, Welding, Space Curve, Control Methodology

\section{Introduction}

With the advantage of high energy density, deep penetration, high precision and strong adaptability, the laser welding as a kind of advanced welding technology is widely used in machining, automobile, steel, medical and other industrial fields. In general, the laser welding has higher productivity and better quality than traditional welding methods. However, there are lots of problems in the welding process of car body parts, such as curve welding seam, low assembly precision, large gap in welding area, etc., which were described in reference [1]. In the process of complex curved surface parts welding, the laser welding head should keep a constant distance to the surface of the sheet in order to eliminate the gap between the plates and make the position of the laser beam focus be unchanged for good welding quality. Therefore, a mechanical clamping device fixed directly on a multi-degree of freedom robot manipulator to solve the problem of easy deformation of sheet metal plates should be designed.

In order to solve the space free curve welding problem, welding robot has been widely used in the practical production. Chen [2] presented a novel modeling method for three degrees of freedom curve welding seam based on a welding robot and the curve welding seam tracking is implemented by macro and micro motion to control the translational motion of the torch in a large range. Graaf [3] proposed a real time seam tracking algorithm to cope with the accuracy demands of robotic laser welding. LI [4] developed a kind of plane curve welding to meet the needs of plane curve weld bead. An intersection welding robot was designed [5] to meet the special welding requirements of intersecting pipes. ZHANG [6] designed an automatic welding and cutting equipment for the space curve trajectory welding cutting. The device can roll freely in the free curve path and maintain the space curve trajectory of the torch at the best position. LI [7] designed the 
welding equipment equipped upon an auto-welding machine which can equally distribute among the welding seam. GAO [8] studied the curved seam welding problem in ship building and large spherical tank welding and provided the research direction to design the clamping device. For the problem of compact the space free curved surface sheet metal in welding process, WANG [9] described the recent achievements in the development of computer aided fixture design methodologies, systems and applications. TADIC [10] focused on the problem of compliance of interface between clamping/locating fixture elements and workpiece during machining. Shikoda [11] put forward a complex laser-welding head that has capable of applying a fixed pressure to work pieces regardless of the undulation. Devrient [12] proposed a dual clamping device (DCD) to prevent the risk of downtime or poor weld seam quality. DENG [13] designed an adaptive fixture which could press the surface of part in constant stress. LIU [14] design a compacting device based on distributed elastic springs to obtain the best fixture result.

In order to solve the problem of easy deformation of sheet metal plates in three dimensions space, a mechanical clamping device, which can be fixed directly on a multidegree of freedom robot manipulator, is investigated in this paper. Thanks to the proposed modular construction and the use of a conventional spring, the device shows good performances. The developed clamping device can also be extended to other applications in surface manufacturing such as spray painting on space free curve.

\section{Conceptual Design}

The aim of this section is to descript the kinematic diagram of the clamping device. In order to solve the problem of compacting sheet plates and moving on space free surface in the process of laser welding, a mechanical clamping device fixed directly on a multidegree of freedom robot manipulator is designed. The device is mainly composed of a linear motion device, a spring device, a pressing wheel device and the control system. The kinematic diagram of the device is shown in Figure 2.

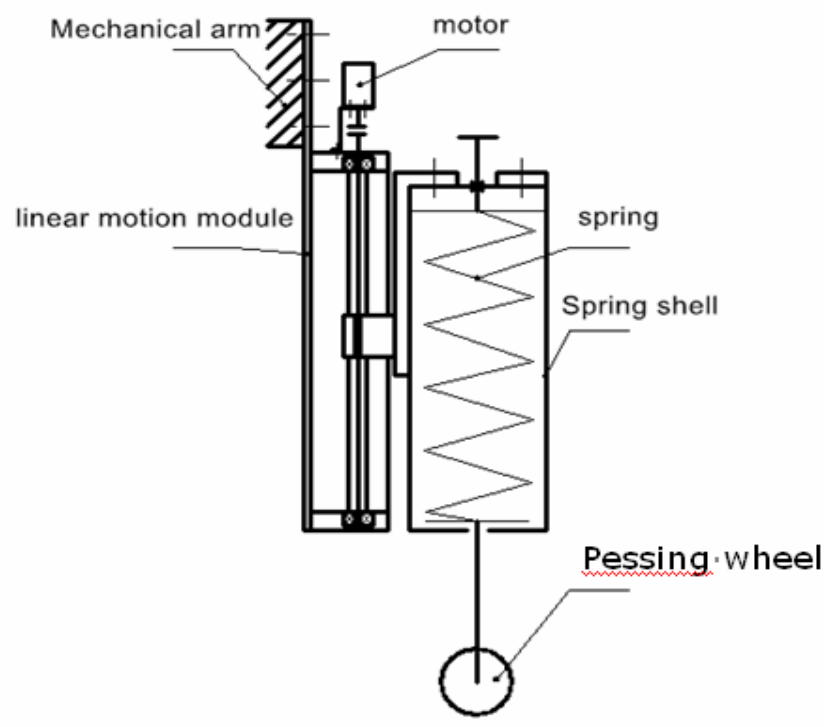

Figure 2. The Kinematic Diagram of the Clamping Device

\section{Mechanical Design}

\subsection{Mechanical Structure}

In this section, the mechanical design of the clamping device will be analyzed. According to the kinematic diagram of the clamping device, the mechanical structure 
design of the clamping device was performed using a 3D CAD system (SolidWorks) is shown in Figure 3.

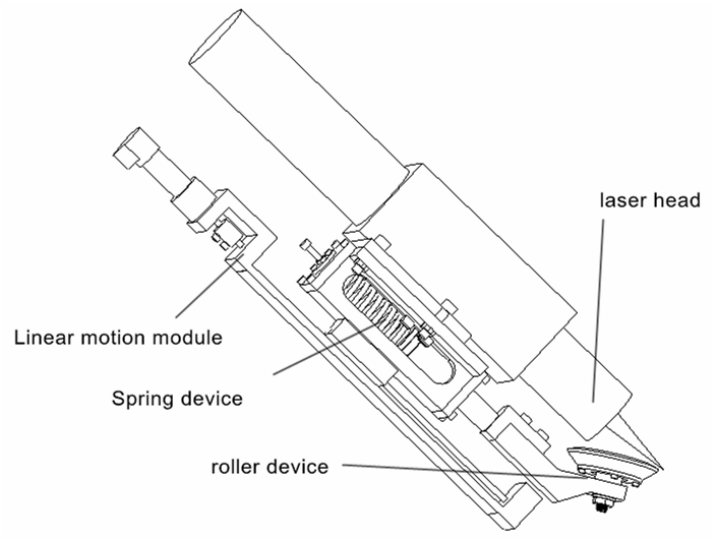

\section{Figure 3. Three-dimensional Assembly Mechanical Model}

The function of the pressing wheel device is to provide the clamping force to sheet plates through a pressing wheel rolling on the surface of sheet plates, which is generated by the compressed spring. The design of the clamping force will change with the compressed length of the spring. The spring device, connecting the pressing wheel device with the linear module, includes a spring, spring shell and two position sensors. The position sensors are used to indicate the positions of the compressed spring. By the position sensors, we can indirectly infer the maximum and minimum clamping forces and transmit signals to the control system, which will control the motor of the linear motion module to turn in positive or inversion. On one hand, the linear motion module provides a connection to the multi-degree of freedom industrial robots. On the other hand, it also provides necessary connection and support for the components of the clamping device, whose main bearing structure stiffness will become lowest at a certain position along with feed motion. The region of compact force of the spring device is designed from $50 \mathrm{~N}$ to $500 \mathrm{~N}$, which can basically meet requirements in the actual laser welding. Certainly, the region can be changed through changing the pressed spring length.

\subsection{Spring Device Design}

The spring is the key section in the mechanical structure design stage of the proposed clamping device and the detail design process of the spring will be given in this section. In the mechanical design stage of the clamping device, the spring device generates a clamping force on the plate to decrease the welding gap between the plates into the allowable range. So a reasonable design of the spring device is needed to meet the requirements of the adjustable pressure.

In the mechanical design stage of the clamping device, the spring device provides the clamping force to decrease the gap in an allowable range. So a reasonable design of the spring device is needed. Let the spring wire diameter to be $3.5 \mathrm{~mm}$ in advance. In order to verify the spring wire diameter, some calculations are necessary. The spring wire diameter should meet the constraint as follows:

$$
d \geq 1.6 \sqrt{\frac{K F_{\max } C}{\tau}}
$$

where $\mathrm{d}$ denotes the spring wire diameter, $K$ denotes the curvature correction coefficient, and

$$
K=\frac{4 C-1}{4 C-4}+\frac{0.615}{C}
$$


$C$ denotes the spring index. In order to ensure the adequate stiffness, where $C=6 . F_{\max }$ denotes the maximum working force of the spring. Let the Hooker coefficient of the spring be

$k=20 \mathrm{~N} / \mathrm{mm}$, the clamping distance of the spring be $20 \mathrm{~mm}$. So $F_{\max }=400 \mathrm{~N}$. The spring is carbon steel $1 \mathrm{Cr} 18 \mathrm{Ni}$ 9Ti. $\tau$ denotes the permissible cut stress and $\tau$ equals $432 \mathrm{MPa}$.

Submitting the data above into (1), we obtain

$$
d \geq 1.33 \mathrm{~mm}
$$

Based on (3), it is reasonable that the spring wire diameter equals $3.5 \mathrm{~mm}$. The pitch diameter of the spring can be determined as

$$
D=C d+d=24.5 \mathrm{~mm}
$$

where $D$ denotes the pitch diameter of the spring. By (4), Let $D$ equals $25 \mathrm{~mm}$ in integer.

More standard machine design formulas for the embodiment of the spring were considered in Ref. [16].

In order to press the sheet plates effectively, it should be nicely guaranteed that the clamping device perpendicular to the surface of welded sheet metal in the process of welding and the spring should avoid bending. The spring device is designed specially and contains a rolling spline pair and a special cylindrical space inside of the spring shell. Rolling spline pair is used to transfer the force of pressure wheel to the spring end cover and limit spring's free rotation to ensure the spring perpendicular to the stress surface. The internal design of spring shell is cylindrical space which is little larger than the outer diameter of the spring. The installation of the spring is shown in Figure 4.

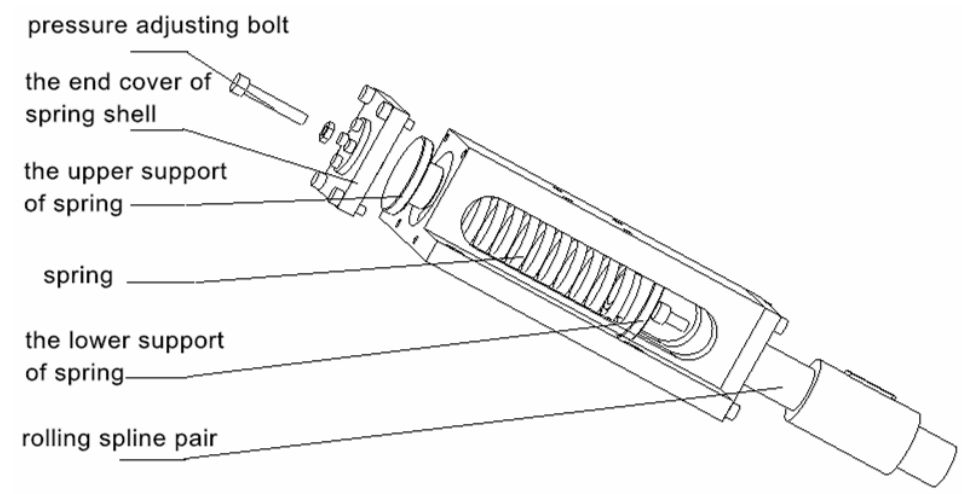

Figure 4. The Installation of the Spring

Do to the sheet plates have many kinds with different material and thickness, causing the requirement of the compact force to be different in the different process of welding. It is required to improve the application range of clamping device and the designed compact pressure of the device to be adjustable.

The bottom of the spring will be compressed to a set position. Let the amount of spring compression to be $\triangle X$, the compact force on the sheet plate by pressing wheel can be obtained

$$
F=F_{0}+k \Delta X
$$

where $F$ denotes the compact pressure on the sheet plate and $F_{0}$ denotes the preestablished mechanical pressure.

\section{Control Methodology}

The control methodology is very important for control system design of the clamping device, which ensures all components to move in order in the process of welding. The 
control system is mainly to receive the signals comes from the pressure sensors and drive the slider and the spring shell moves up or down to keep the constant pressure on the sheet plate. By the open motion control platform, the control software can be developed to achieve real-time control in the welding process according to the control methodology.

When the clamping device moves on space free curved face, the signals obtained from the position sensor in real time will make the motor frequently reverse. To avoid this problem, an allowable displacement is given. According to Hooker's law, the pressure on the plate is obtained:

$$
F_{0}-k \Delta \delta \leq F_{p} \leq F_{0}+k \Delta \delta
$$

where, $F_{p}$ denotes the working pressure and $F_{0}$ denotes the initial set pressure.

Based on (7), the minimum and maximum pressure can be get, respectively:

$$
\begin{aligned}
& F_{\text {min }}=F_{s}-k \Delta \delta \\
& F_{\text {max }}=F_{S}+k \Delta \delta
\end{aligned}
$$

The flow diagram of the control methodology is shown in Figure 5.

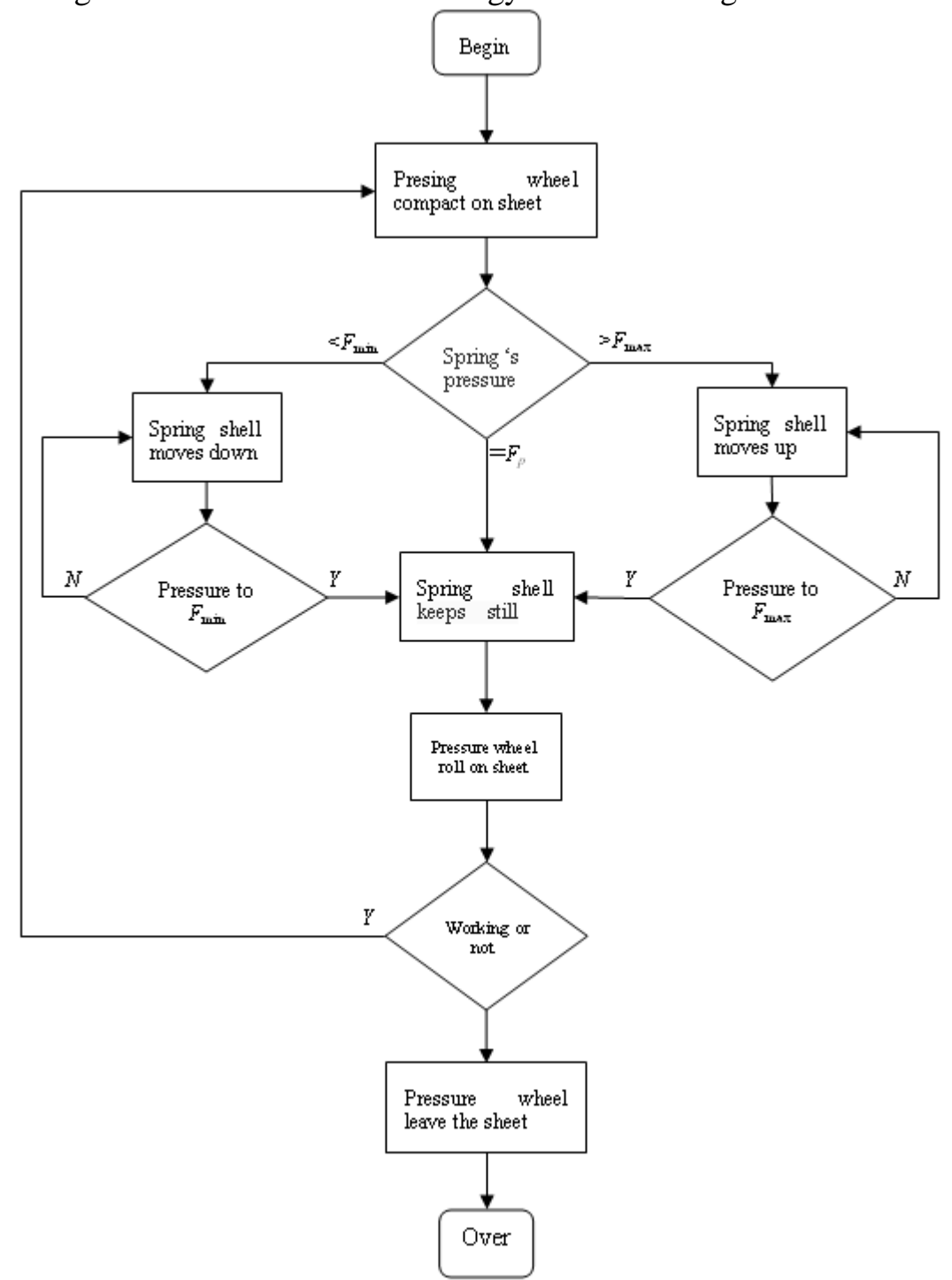

Figure 5. The Control Methodology of the Device 


\section{Conclusion}

In this paper an adaptive clamping device for the laser welding on space free curved face is designed, which has the advantage of simple structure, adjustable compact force and low cost, etc. The conceptual design, the mechanical design, and the design of control system of a new mechanical clamping device are introduced in detail. The clamping device which is mounted on welding robot (or welding machine) manipulator can not only to complete the compact of space free curved face sheet metal, but also to keep a constant distance between the laser head and the welding area in the laser welding process. The clamping device might improve the labor productivity and the welding quality significantly, and the labor intensity be reduced. Thanks to the proposed modular construction and the use of a conventional spring, the device shows good performances. The developed clamping device can also be extended to other applications in surface manufacturing such as spray painting on space free curve.

\section{ACKNOWLEDGEMENTS}

This work is partially supported by National Science Foundation of PR China (51105003), Beijing Postdoctoral Research Foundation (PHR200906107), Excellent Young Teachers Training Program of NCUT (14085), and Scientific Research Common Program of Beijing Municipal Commission of Education (KM201110009010).

\section{References}

[1]. L. Mei, Editor, "Study on Three-dimensional Laser Cutting and Lap-welding of Auto body-in-white part", Hunan University, (2010).

[2]. H. Chen, D. Xu and H. Wang, "Three Dimension Curve Welding Seam Modeling for Seam Tracking", Proceedings of the 17th World Congress of the International Federation of Automatic Control, (2008) July 6-11, Seoul, Korea.

[3]. D. G. Menno, A. Ronald, J. Ben and M. Johan, "Real-time seam tracking for robotic laser welding using trajectory-based control", Control Engineering Practice, vol. 18, (2010), pp. 944-953.

[4]. R. Li, "Design and Realization of 3-DOF Welding Manipulator Control System Based on Motion Controller", Energy Procedia, vol. 14, (2012), pp. 931-936.

[5]. F. Ren, S. Chen, X. Guan and S. Yin, "Special purpose welding robot for intersection welding seam", Trans. of the China Welding Institution, vol. 30, (2009), pp. 59-62.

[6]. Z. Zhang, "Design of Automatic Welding and Cutting Equipment for Space Curve Trajectory", Welding Technology, vol. 24, (1995), pp. 11-12.

[7]. C. Li and Q. Geng, "Design of special automatic welding equipment", Electric Welding Machine, vol. 38, (2008), pp. 61-64

[8]. Y. Gao, H. Zhang, J. Peng and Z. Mao, "Curved Seam Tracking Control of a Wheeled Welding Mobile Robot”, Robot, vol. 29, (2007), pp. 439-442,450.

[9]. H. Wang, Y. Rong, H. Li and P. Shaun, "Computer aided fixture design: Recent research and trends", Computer-Aided Design, vol. 42, (2010), pp. 1085-1094.

[10].T. Branko, V. Djordje and M. Dragomir, "Model testing of fixture-workpiece interface compliance in dynamic conditions", Journal of Manufacturing Systems, vol. 33, (2014), pp. 76-83.

[11].S. Shikoda, T. Kubota, H. Uehara, O. Satoh, T. Uno, T. Murata, and M. Nishio, "Laser Welding Head with Associated Roller", U.S. Patent 6,633,018,B2, (2003) October 14.

[12].M. Devrient, B. Knoll and R.Geiger, "Laser transmission welding of thermoplastics with dual clamping devices”, Physics Procedia, vol. 41, (2013), pp. 70-80.

[13].H. Deng, "Design of Adaptive Fixture for Clamping of Free Form Components", Machine Tool \& Hydraulics, vol. 40, (2013), pp. 67-69, 66.

[14].G. Liu, Z. Feng, M. Zhang and Y. Ke, "Design of Compacting and Iron Powder Filling Device in Paper Honeycomb Parts Clamping System", Acta Aeronautica et Astronautica Sinica., vol. 33, (2012), pp. 1938-1946.

[15].L. Xin, Z. Xu, M. Zhao and Y. Zou, "Study and Exploitation on a Novel Laser Stitch Welding System", Journal of Mechanical Engineering, vol. 45, (2009), pp. 219-225.

[16].J. Shigley, C. Mischke and T. Brown, "Standard Handbook of Machine Design", Columbus, OH, McGraw-Hill, (2004). 